\title{
Promoting Larval Source Management as a Vital Supplemental Addendum and More Likely Cost-Effective Approach for Malaria Vector Control in Nigeria
}

\author{
Oluwasogo A Olalubi ${ }^{1,2}$ and Godson K Chinwe ${ }^{1,3}$
}

${ }^{1}$ Centre for Ecological and Environmental Research Management Studies, Kwara State University, Nigeria

${ }^{2}$ School of Allied Health and Environmental Sciences, Department of Public Health, Kwara State University, Nigeria

${ }^{3}$ National Malaria Elimination Programme, Federal Ministry of Health, Abuja, Nigeria

Corresponding author: Oluwasogo A Olalubi, School of Allied Health \& Environmental Sciences, Department of Public Health, Kwara State University, Nigeria, Tel: +234-703-603-9078; Email: Olalubisogo@gmail.com

Received date: May 31, 2016; Accepted date: July 18, 2016; Published date: July 25, 2016

Citation: Olalubi OA, Chinwe GK. Promoting Larval Source Management as a Vital Supplemental Addendum and More Likely Cost-Effective Approach for Malaria Vector Control in Nigeria. Prev Inf Cntrl. 2016, 2:2.

Copyright: (c) 2016 Olalubi OA, et al. This is an open-access article distributed under the terms of the Creative Commons Attribution License, which permits unrestricted use, distribution, and reproduction in any medium, provided the original author and source are credited.

\section{Abstract}

Malaria is an important cause of morbidity and mortality in people living in many parts of the world, especially SubSaharan Africa and particularly Nigeria. Nigeria has a vision to become malaria-free with a mission to liberate the populace from the scourge of malaria-driven morbidity, mortality and impoverishment through effective leadership, integrated health system strengthening and intersectoral coordination. Larval source management (LSM) refers to the targeted management of mosquito breeding sites, with the objective to reduce the number of mosquito larvae and pupae in the environment. However, very little or no attention has been given to this very important malaria vector control intervention especially in Nigeria. The WHO position statement stated that for effectiveness, larviciding must be specially adapted to each locality, and must be carried out thoroughly and selectively, and should be considered for malaria control with or without other interventions only in areas where the breeding sites are few, fixed and findable. The National Malaria Elimination Programme in collaboration with its implementing partners have carried out series of field efficacy trials of different strains of larvicides to ascertain the effectiveness of the intervention as well as its feasible capabilities to reduce the malaria vector population density and has given a node for the programmatic deployment of the intervention. In the early twentieth century larviciding and environmental management were the only tools available to contain malaria. Historical literature and more recent reviews of this approach show that anti-larval mosquito control measures were powerful tools against malaria. One of the key challenges for conventional and microbial larvicides used for malaria control in Africa is the short residual period of control. However, LSM is a well-established strategy, it is a tool ready for use without any further research required. If the current dream of malaria elimination is to be realized, LSM in combination with Long-Lasting Insecticide Treated BedNets (LLITNs) and Indoor Residual Spraying (IRS) remains the practicable fast train that will take us to our destination of becoming a malaria-free country.

\section{Keywords}

Larval source management; National malaria elimination programme; World Health Organization; Breeding sites; Larvicides

\section{Introduction}

Majority of the world's population live in areas at risk of vector borne diseases, most of which are spread through mosquitoes [1]. There is no gain saying the fact that recent estimates suggest that malaria mortality rates decreased by an impressive $47 \%$ between 2000 and 2013 globally and by $54 \%$ in the World Health Organization's (WHO) African Region, nevertheless, malaria still remains a major public health problem in a number of countries [2]. Emphatically, Malaria is transmitted throughout Nigeria, with $97 \%$ of the population at risk and the country loses about $\mathrm{N} 480 \mathrm{~b}$ annually on malaria prevention and treatment. Five ecological zones define the intensity and seasonality of transmission and mosquito vector species: mangrove swamps, rain forest, Guinea-savannah, Sudan-savannah and Sahel-savannah. The duration of the transmission season decreases from year-round transmission in the south to three months or less in the north. Plasmodium falciparum is the predominant malaria species. The major vectors are Anopheles (An.) gambiae and An. funestus. Within the An. gambiae complex, An. arabiensis predominates in the north and An. melas in the mangrove coastal zones [3].

Nigeria has a vision to become a malaria-free country with a mission to free every Nigerian citizen from the scourge of malaria through effective leadership, environmental sanitation, health system strengthening and coordination in developing efficient policies, strategies and guidelines [4]. In the pursuit of the above, the National Malaria Elimination Programme since the global declaration of Roll Back Malaria in year 2000 has scaled up evidence-based vector control interventions for impact. Certain achievements have been recorded and these 
include the reduction of current slide positivity rate for children under five (5) years to $42 \%$ (MIS, 2010), under five (5) years mortality has reduced from 201/1000 in 2003 to 157/1000 in 2008 and 128/1000 in 2013, Ante Natal Care (ANC) attendance has also increased from $58 \%$ in 2008 to $61 \%$ in 2013 [5].

Vector control is an essential component of malaria prevention. Such control has been proven to successfully reduce or interrupt malaria transmission when coverage is sufficiently high. Long Lasting Insecticidal Nets (LLINs) and Indoor Residual Spraying (IRS) have been found to reduce malaria transmission by targeting the adult mosquito vector which is a key component of malaria control Programme. However, unpredictable mosquito larvae and pupae population transition dynamics, especially during the favourable transmission seasons still remains a critical issue in the tropics. Ability to carefully address and tame this subtle indicator would eventually determine the level of success attainable by the malaria elimination agenda. This should be significantly reduced through correct, consistent and effective application of Larval Source Management (LSM), which targets mosquito larvae as they mature in aquatic habitats. Larval source management (LSM) refers to the targeted management and control of mosquito breeding sites, with the objective to reduce the number of mosquito larvae and pupae. There are four main types of LSM: (1) habitat modification, which means a permanent alteration to the environment, e.g. land reclamation or surface water drainage; (2) habitat manipulation, which refers to a recurrent activity e.g. water-level manipulation, flushing of streams, the shading or exposure of habitats; (3) larviciding, which involves the regular application of a biological or chemical insecticide to water bodies; and (4) biological control, which refers to the introduction of natural predators into water bodies, for example predatory fish or invertebrates [6].

However, very little or no attention has been given to this very important malaria vector control intervention especially in Nigeria.

This review addresses high demand for prospect, role, strength efficiency and accountability for maximum impact as well as the gross neglect or under-utilization and challenges of Larval Source Management as an important strategy for malaria vector control in Nigeria despite past success stories of this intervention in contributing to the control and or elimination of malaria in many countries. The objective of this paper is to appraise relevant literatures on the global perspective, success stories and feasible capabilities of LSM as an important tool that would contribute to the attainment of the current malaria elimination goal of the Federal Government of Nigeria.

Role, benefit, strength, global perspective of larval source management in malaria control and elimination

Anopheles larvae are 'sitting ducks'; they are relatively immobile and often readily accessible. By targeting the larval stages, mosquitoes are killed 'whole sale' before they disperse to human habitations. Mosquito larvae, unlike adults, cannot change their habitat to avoid control activities [7]. The elimination of aquatic habitats close to human habitations by environmental modifications and manipulations, where possible, can provide long-term and cost-effective solutions. Once a habitat is gone it does not produce any flying and biting mosquitoes [8]. This is particularly true in urban areas where drainage of aquatic habitats can be incorporated into on-going town or city development plans [9]. In many cases these costs will be paid outside the health sector. In places where habitats cannot be eliminated, a number of very effective larvicides are available that reduce mosquito production rapidly. There are a broad range of effective formulations that have been developed for anopheline control [10]. The diverse family of larvicides provide a wide range of modes of actions against Anopheles larvae including microbials that lyse the gut epithelium, insect growth regulators that prevent the larvae developing into adults, synthetic or botanical toxins that directly interfere with the insects' metabolism and monolayers that lead to suffocation of larvae. Today's larvicides are environmentally acceptable with minimal or no effect on non-target invertebrate populations, aquatic ecosystems, beneficiary insects, fish, birds and mammals, including humans. Larviciding requires no substantial change in human behaviour or the management of key resources such as water and land, and skills for larviciding can be similarly acquired as those for IRS [11]. When appropriately and effectively used, LSM contribute to reducing the numbers of both indoor and outdoor biting mosquitoes, and in malaria elimination phase, it can be a useful addition to programme tools to reduce the mosquito population in remaining malaria 'hotspots'. Where appropriate, it can also help programmes to reduce their overall dependence on insecticides, thereby making a contribution to preventing the emergence of insecticide resistance. LSM can also be useful to help control other vectorborne diseases, especially Dengue and Zika [6]. LSM is an approach that needs to be tailored to local environmental conditions. LSM programme design must be appropriate to the local infrastructure. In general, there are various approaches to implementing LSM:

LSM can be built into the national malaria control programme, in which case any country considering LSM should start on a small scale with pilot schemes and then build capacity and experience. In this context, LSM requires more than the current funding and political support: strategic, long term funding is needed so that local programmes and supporting institutions have time to learn and consolidate.

Small communities or municipalities with few resources but significant motivation to control malaria, such as in places where LLINs and/or IRS have not yet been deployed, can conduct LSM as part of a local community effort.

Sectors outside the health sector can contribute support to LSM through careful road and building construction and infrastructure development, as practiced in Khartoum [6]. Large urban areas and private schemes such as mining and agricultural operations, with an interest in malaria control and improved quality of life through reduction in nuisance mosquito populations, can implement LSM independent of, but in collaboration with, national malarial control activities using local or corporate resources [6]. 
LSM has an advantage of abating general mosquito population rather than anopheline control alone. This should generate more support for the programme from the local population and at the same time produce infrastructure and reinforcement for the control of culicine, aedes mosquitoes especially where Zika and allied arboviruses are potential public health problems.

Engaging LSM in urban and Peri-Urban areas: Larval habitats in towns and cities are largely man-made and relatively easy to identify and treat, as seen in Khartoum and Dar es Salaam. In addition, other interventions, which may be acceptable to a rural population, may not be well received in urban areas, such as IRS in India, where LSM is the mainstay of urban malaria vector control. In Zanzibar, where IRS as deemed not feasible in the urban area of Stone town, due to the density and structure of houses coupled with very low levels of malaria transmission, the malaria programme decided to plan for larviciding in this area.

\section{Contributory factors of success of LSM programmes in malaria control and elimination}

Community acceptance, mobilization, involvement and support: LSM interventions should strive towards gaining the support of the local community in a target area so that larval habitats can be accessed and either treated with a larvicide or modified. Local needs must be taken into consideration when interventions are planned, e.g. the livelihood of the local population might depend on some of the aquatic habitats (rice fields, irrigation channels and pits, wells). Therefore, educational programmes need to be implemented prior to interventions and ideally community members should be directly involved in conducting LSM, as seen in Khartoum, Mauritius and Dar es Salaam [6].

Effective leadership, good management and clarity of objectives: Personnel at all levels of the health system must receive the message that $L S M$ is an important undertaking and has the support of the management. Management capacity development is the key to a successful LSM programme. Of particular importance is the ability to quickly generate, collate, report meaningful monitoring data. In reality, inadequate training and management of staff and logistics could limit the success of LSM programmes in any suitable areas.

The need for Entomologists with detailed knowledge of local vectors: It is imperative to have trained entomologists who conduct detailed surveys of the ecology and behaviour of local vectors, in order to establish which (if any) LSM interventions are appropriate and to monitor the impact of the programme [6].

Promotion of Collaboration between sectors: LSM often overlaps with the responsibilities of other sectors and therefore careful coordination can be productive and reduce costs. For example, since 2002 the Malaria Free Initiative in Khartoum State, Sudan, has coordinated with the Public Works Department (to repair broken water pipes which are an important source of vector larval habitats), the Farmers' Union and the Ministry of Agriculture (to promote intermittent irrigation), the Ministry of Education (to involve school children directly in LSM) and the media (to increase radio and television broadcasts to raise public awareness and support for the campaign). Collaboration with other sectors to ensure good practice in infrastructure development and housing is also important, so that activities such as road construction, brick making or house building do not create new larval habitats [6].

The need to build strong surveillance systems: Continuous entomological monitoring is crucial to ensure that all larval habitats are being correctly handled, and epidemiological surveillance is important to monitor the impact of the LSM programme.

\section{Management, frequency of application and cost effectiveness of LSM for malaria vector control and elimination}

The WHO states that in order to be effective, larviciding must be specially adapted to each locality, and must be carried out thoroughly and selectively. In general, larviciding should be considered for malaria control with or without other interventions in areas where the breeding sites are few, fixed and findable [12]. Applying larvicides to all potential breeding sites can be cost effective in urban centres. The current strategy of LSM with larvicides is to treat all available larval habitats [13]. Some argue for a more spatially targeted approach [14] to apply larvicides only at the most productive habitats [15]. At present though we still lack scalable field methods for determining which habitat subsets are the productive ones. In fact to date no published evidence exists that shows that accurately determining where malaria vectors will develop is possible [16]. There is both spatial and temporal variation in the distribution of Anopheles larvae. Whilst some types of habitats are more likely than others to have aquatic stages [17], this is not sufficiently refined for spray personnel to be able to identify and target only these high-risk habitats. Most importantly, when it comes to the implementation of LSM, treatment of all sites is much easier for field personnel since this requires minimum decision making and is, therefore, less prone to mistakes [18]. However, several models have been developed recently to predict mosquito larval habitats location and productive potential, so in future it may well be possible to target interventions more effectively [19]. Any benefit of targeting larval habitats at specific times of the year needs to be proven, but may work well when LSM is part of an IVM package of interventions [19]. Thus, in the future, LSM may be targeted in space, when 'hotspots' of transmission have been identified, or in time, to restrict biting densities at certain times of the year [20]. In both cases the scale of the intervention would be considerably smaller than the routine application of blanket larviciding.

Another concern is the application frequency of larvicides. At present microbial larvicides are generally applied weekly to all potential sites [18]. Whilst larvicides with greater residual activity would be beneficial for treating permanent habitats [21], it is important to note that they are not necessarily the panacea they might appear to be since during periods of rain new potential mosquito larval habitats can appear and larvae can 
develop into adults before the next round of application. Thus where sites are actively dynamic, weekly application is more effective because new sites are treated promptly and it is simpler because the people who apply the larvicide become familiar with their treatment area and the weekly cycle of activity. Overall, targeting interventions in space and time as well as the use of more residual larvicides will only reduce costs if proven to be equally effective than blanket application and if the increased management effort for decision making does not outweigh the larvicide costs [22]. Nonetheless, substantial reductions in long-term costs may be made if larviciding is combined with environmental management. A recent study in Dar es Salaam demonstrated that simply by improving drainage in drains would reduce larval breeding by $40 \%$ [9]. Since malaria is a problem created by surface water, it is still surprising that engineers are rarely engaged in malaria control [23] since there are many simple and effective engineering solutions to reduce mosquito larval habitats [19].

\section{Feasible capabilities of LSM as an important tool for malaria vector control and elimination}

Today there is renewed interest in LSM [18] and its practical application in Africa as a complementary intervention to LLINs and IRS, especially where outdoor biting by malaria vectors is problematic or where there is resistance to the insecticides used for LLINs or IRS [18]. Field trials in different eco-epidemiological settings in Africa and Asia (where larval habitats were few, fixed and findable) have shown that larviciding can reduce the density of adult vectors and consequently malaria transmission and morbidity [6].

The National Malaria Elimination Programme in collaboration with its implementing partners carried out series of field efficacy trials of different strains of larvicides to ascertain the effectiveness of the intervention as well as its feasible capabilities to reduce the malaria vector population density. The Programme during one of its field trial of Skeeter ABATE 5\% PG (Tempos), identified mosquito larval control as a potential IVM tool that aid in the reduction of malaria transmission. The residual efficacy of Skeeter ABATE 5\% PG was evaluated in Lagos and Jigawa States of Nigeria. The evaluation took place in three Local Governments of Lagos State namely, Ojo, Badagry and Ikorodu. Badagry and Ikorodu LGAs sites contained predominantly anopheline and culicine mosquitoes. The two sites in Ojo LGA have culicine only. The larvicide was applied by hand and with a motorized back pack with a granular applicator. It was applied at a rate of $11.21 \mathrm{~kg} / \mathrm{ha}$ by trained malaria control personnel from Ojo LGA. The result revealed that the larvicide performed excellently well and provided effective anopheles control for 30 days. The residual component and the "Prehatch" characteristic of this formulation will allow malaria control programmes more flexibility in the larviciding programme for malaria control and provide protection for a large population of people who do not always comply with other mosquito control interventions such as the use of ITNs or IRS [24]. Another field trial was conducted by the NMEP on mosquitocidal strains of Bacillus thuringiensis var. israelensis (Bti) and Bacillus sphaericus (Bs) in Ikenne LGA of Ogun State in February 2011. The result revealed that the biological larvicides were highly effective against all strains of Anopheline, culecine and aedes mosquitoes [25]. The third trial was conducted in Ogun and Nasarawa States on another formulation of Bacillus thuringiensis isrealensis serotype H-14 (BACTIVEC) and Bacillus sphaericus strain 2362 (Griselesf). Effectiveness and residual efficacy of bactivec and griselesf biolarvicides were proven for the control of anopheles and other species present such as the Culex quinquefasciatus. In the treated breeding sites $(1,2$ and 4$)$ a stable and significant reduction was observed from the first $24 \mathrm{hr}$ to the $30^{\text {th }}$ day in at least 3 of the 4 treated sites within the ranges of 80.3 to 100 percents (NMEP report of field trial on Bacillus thuringiensis isrealensis serotype H-14 and Bacillus sphsaericus strain 2362, 2013) [26].

\section{Past success stories of LSM as a tool for the control of malaria vector}

Today there are 734 named mosquito abatement districts in the US, all deploying LSM, which is the primary and preferred method of mosquito control in the US. LSM is practiced in California and Florida, often controlling mosquitoes that occur on far more prodigious scales than found in Africa. In the largest district, Lee County Florida, the annual budget for mosquito control exceeds $\$ 19 \mathrm{~m}$, whilst in the Metropolitan Mosquito Abatement District the budget is over $\$ 18 \mathrm{~m} \mathrm{[27].} \mathrm{In} \mathrm{the} \mathrm{60s,}$ Nigeria embarked on the deployment of LSM for malaria vector control where Environmental Health Officers were referred to as Health inspectors whose responsibilities included larviciding of standing waters, fumigation of drainage systems, inspection of residential and business premises to ensure the elimination of mosquito breeding sites through simple environmental sanitation. This strategy led to the reduction of morbidity and mortality due to malaria and improved the health status of the people. In the early twentieth century larviciding and environmental management were the only tools available to contain malaria. The historical literature and more recent reviews of this approach show that anti-larval mosquito control measures were powerful tools against malaria. First report of anti-larval measures used for malaria control in Africa was in Freetown, Sierra Leone, in 1812, where there were a law preventing people from allowing stagnant pools which generate disease and mosquitoes over the town. Since then, Anopheles larval control has been a central pillar of many successful malaria control programmes worldwide. More so, the most remarkable achievements with larviciding were the elimination of Anopheles arabiensis, a member of the An. gambiae complex, from Brazil and Egypt. In the 1930s, An. arabiensis, a major vector of malaria in Africa, was introduced accidentally into Brazil resulting in an epidemic that killed thousands of people and turned the countryside into a wilderness. Most remarkably, a larval control programme ran with military precision was able to eradicate $A n$. arabiensis within 2-3 years [28]. These vectors have also been successfully controlled in the heartland of malaria in Africa (Zambia). Malaria was a major threat to the economic success of the copper mines in Zambia in the first half of the twentieth century. An integrated malaria vector control programme, primarily based on attacking the larval stages of malaria vectors 
by environmental management resulted in $97 \%$ reduction of annual malaria incidence from $514 / 1,000$ in $1929 / 1930$ to $16 / 1,000$ in $1949 / 1950$. Similarly, overall mortality fell by $88 \%$ from 32/1,000/year to 4/1,000/year [29].

\section{Challenges of LSM}

One of the key challenges for conventional and microbial larvicides used for malaria control in Africa is the short residual period of control. This short time frame requires control programmes to treat habitats and conduct surveillance on a weekly basis. Cost to maintain these programmes in personnel and product are extremely high and limit the use of larvicides to a few well-funded programmes.

The safety and efficacy of larval control products is of critical importance. Within WHO, it is the WHO Pesticide Evaluation Scheme (WHOPES) that coordinates the assessment of larval control compounds and formulations. WHO encourages endemic countries to procure WHOPES recommended products; the use of non-recommended products may have adverse impacts on human health and the environment.

\section{Conclusion}

Mosquito larval control will work best and be most costeffective in areas where larval habitats are well-defined possibly seasonal or relatively few, where habitats are accessible by ground crews, and in cooler parts of Africa where larval development is prolonged. These conditions occur frequently, even in sub-Saharan Africa, and thus this method can be an effective tool for malaria control in selected eco-epidemiological conditions such as areas of low to medium transmission intensity, areas of focal transmission or epidemic prone areas. Such conditions are common in urban environments, desert fringe communities, highland settlements and rural areas with high population densities. Recent field evaluations under various eco-epidemiological conditions in Africa showed that handapplied larviciding reduced malaria transmission by $70-90 \%$ where the majority of aquatic mosquito larval habitats were defined and aquatic surface areas not too extensive. However, the enhanced holistic approach still involved the rationale use of combination of environmental management and larviciding which could be conducted by temporarily or permanently reducing the availability of larval habitat (habitat modification and manipulation), or by adding chemical substance to standing water that either kill or inhibit the development of larvae (Larviciding). Addition of larviciding with LLINs and IRS resulted in greater gains than could be achieved by using LLINs or IRS alone as currently being practiced in Nigeria. LSM is a wellestablished strategy, it is a tool ready to use without any further research required. If the current dream of malaria elimination in Africa and Nigeria in particular is to be realized, LSM in combination with LLINs and IRS remains the fast train that will take us to our destination of becoming a malaria-free country.

\section{Acknowledgement}

We appreciated Dr. Nnenna Ezeigwe, the National Coordinator, NMEP Nigeria and Dr. Joel Akilah, Head of IVM Unit for their technical support.

\section{Competing Interests}

The authors declare no conflict of interest.

\section{Authors' Contribution}

GKC conceived the idea and submitted the initial draft of the manuscript. OAO restructured the concept and develop the manuscript, gathered and selected useful publications, provided critical technical insights and input and supervised the entire work. All authors read and approved the final manuscript.

\section{Funding Information}

The author(s) declared that no grant was involved to support the work.

\section{References}

1. Guruprasad NM, Jalali SK, Puttaraju HP (2014) Wolbachia-a foe for mosquitoes. Asian Pac J Trop Dis 4: 78-81.

2. Abdoulaye AD, Amelia WM, Dinkorma O, Bakary F, et al. (2016) Gametocyte clearance dynamics following oral artesunate treatment of uncomplicated falciparum malaria in Malian children. Parasite 23: 3

3. President's Malaria Initiative Nigeria, Malaria Operational plan FY (2016).

4. National Malaria Strategic Plan, 2014-2020.

5. National Demographic Health Survey (NDHS) (2010).

6. WHO (2013) Larval source managememt: A supplementary measure for malaria vector control, An operational manual.

7. Killeen GF, Fillinger U, Knols BGJ (2002) Advantages of larva control for African malaria vectors: Low mobility and behavioural 
responsiveness of immature mosquito stages allow high effective coverage. Malar J 1: 1-8.

8. Keiser J, Singer BH, Utzinger J (2005) Reducing the burden of malaria in different eco-epidemiological settings with environmental management: A systematic review. Lancet Infect Dis 5: 695-708.

9. Castro MC, Kanamori S, Kannady K, Mkude S, Killeen G, et al. (2010) The importance of drains for the larval development of lymphatic filariasis and malaria vectors in Dar es Salaam, United Republic of Tanzania. PLoS Neglect Trop Dis 4: e693.

10. WHO (2006) Pesticides and their application for the control of vectors and pest of public health importance 6 , Geneva.

11. Fillinger U, Ndenga B, Githeko A, Lindsay SW (2009) Integrated malaria vector control with microbial larvicides and insecticidetreated nets in western Kenya: A controlled trial. Bull World Health Organ 87: 655-665.

12. WHO (2010) Handbook on Integrated Vector Management (IVM) Geneva: World Health Organization.

13. Majambere $S$, Pinder M, Fillinger $U$, Ameh $D$, Conway $D J$, et al. (2010) Is mosquito larval source management appropriate for reducing malaria in areas of extensive flood in The Gambia? A cross-over intervention trial. Am J Trop Med Hyg 82: 176-184.

14. Smith D, McKenzie FE, Snow RW, Hay S (2007) Revisiting the basic reproductive number for malaria and its implications for malaria control. PLoS Biol 5: e42.

15. Gu W, Novak R (2005) Habitat-based modeling of impacts of mosquito larval interventions on entomological inoculation rates, incidence and prevalence of malaria. Am J Trop Med Hyg 73: 546-552.

16. Killeen GF, Tanner M, Mukabana WR, Kalongolela MS, Kannady K, et al. (2006) Habitat targeting for controlling aquatic stages of malaria vectors in Africa. Am J Trop Med Hyg 54: 517-518.

17. Fillinger $\mathrm{U}$, Sombroek $\mathrm{H}$, Majambere $\mathrm{S}$, Van Loon E, Takken $\mathrm{W}$, et al. (2009) Identifying the most productive breeding sites for malaria mosquitoes in The Gambia. Malar J 8: 62-100.
18. Fillinger U, Kannady K, William G, Vanek MJ, Dongus S, et al (2008) A tool box for operational mosquito larval control: Preliminary results and early lessons from the Urban Malaria Control Program in Dar es Salaam, Tanzania. Malar J 7: e20.

19. Fillinger U, Lindsay SW (2011) Larval source management for malaria control in Africa: Myths and reality. Malaria Journal 10:353.

20. Worrall E, Fillinger U (2011) Large-scale use of mosquito larval source management for malaria control in Africa: A cost analysis. Malar J 10: 338.

21. Yapabandara AM, Curtis CF (2004) Vectors and malaria transmission in a gem mining area in Sri Lanka. J Vec Ecol 29: 264-276.

22. Parvez SD, Al-Wahaibi SS (2003) Comparison of three larviciding options for malaria vector control. East Mediterr Health J 9:627-636.

23. Beier J, Keating J, Githure J, Macdonald M, Impoinvil D, et al. (2008) Integrated vector management for malaria control. Malar J $7: 54$

24. NMEP report of field trial on Skeeter ABATE 5\% PG (2010).

25. NMEP report of field trial on Bti and Bs (2011).

26. NMEP report of field trial on Bacillus thuringiensis isrealensis serotype H-14 and Bacillus sphaericus strain 2362 (2013).

27. Source reduction in Florida's salt marshes (2006) Management to reduce pesticide use and enhance the resource. J Am Mosq Control Assoc.

28. Eradication of Anopheles gambiae from Brazil (2002) Lessons for malaria control in Africa. Lancet Infect 2: 618-627.

29. Integrated Vector Management: The Zambian experience. Malar $\mathrm{J}(2008)$. 